\title{
Una reconsideración pluralista del concepto de herencia*
}

\section{A pluralistic reconsideration of the concept of inheritance}

\author{
MARIANO MARTÍN-VILLUENDAS \\ Universidad de Salamanca
}

Recibido: 29/09/2020 Aceptado:15/03/2021

\begin{abstract}
RESUMEN
A raíz de los recientes descubrimientos en disciplinas emergentes como la epigenética o la ecodevo, el concepto genocentrista de herencia propio de la Síntesis Moderna ha sido sometido a un intenso análisis y revisión. El presente trabajo tiene por objetivo analizar las inconsistencias conceptuales y prácticas derivadas del concepto clásico de herencia, así como proponer un concepto pluralista e inclusivo. Para ello, se tomará como fenómeno de referencia la herencia epigenética. De estos análisis se concluirá que solo abrazando dicho concepto es posible superar las limitaciones prácticas y conceptuales del modelo de herencia propio de la Síntesis Moderna.

PALABRAS CLAVE

EPIGENÉTICA, EVOLUCIÓN, ECO-DEVO, GENOCENTRISMO
\end{abstract}

\section{ABSTRACT}

Following recent discoveries in emerging disciplines such as epigenetics or eco-devo, the genocentric concept of inheritance proposed by Modern Synthesis has been subjected to intense analysis and revision. The paper aims to analyze the conceptual and practical inconsistencies derived from the classical concept of inheritance, as well as to propose a more pluralistic concept. To this purpose, epigenetic inheritance will be taken as a reference phenomenon. From

* Agradezco los comentarios de Ana Cuevas Badallo, Juan Vicente Mayoral y de los evaluadores de la revista Contrastes.

(C) Contrastes. Revista Internacional de Filosofía, vol. XXVI No3 (2021), pp. 25-47. ISSN: 1136-4076

Departamento de Filosofía, Universidad de Málaga, Facultad de Filosofía y Letras Campus de Teatinos, E-29071 Málaga (España) 
these analyses it will be concluded that only by embracing a pluralistic concept of inheritance is it possible to overcome the practical and conceptual limitations of the inheritance model presented by Modern Synthesis.

KEYWORDS

EPIGENETICS, EVOLUTION, ECO-DEVO, GENOCENTRISM

\section{INTRODUCCIÓN}

El CALIFICATIVo «Síntesis Moderna» fue acuñado en 1942 por Julian Huxley para hacer referencia a la integración conceptual de la teoría de Darwin con los principios de la genética mendeliana. En la actualidad, un gran número de biólogos han abogado por una revisión y extensión de los principios fundamentales que subyacen a la Síntesis Moderna (Huneman y Walsh, 2017; Pigliucci y Müller, 2010). Este programa de investigación se conoce popularmente como Síntesis Evolutiva Extendida (EES, según su forma en inglés). Si bien las caracterizaciones teóricas de los proponentes de la EES se extienden a la discusión de conceptos tan relevantes como los de plasticidad fenotípica (West-Eberhard, 2003), capacitancia evolutiva evolvability - (Wagner y Altenberg, 1996) o construcción del nicho (OdlingSmee et al., 2003), conceptos que, a juicio de los proponentes de la EES, no se hallan insertos dentro del marco de la Síntesis Moderna, en este artículo nos centraremos únicamente en un concepto clave, el de herencia, y la relación de este con la emergente disciplina de la epigenética.

El objetivo fundamental del trabajo consistirá en llevar a cabo una reformulación del concepto clásico de herencia propio de la Síntesis Moderna. Para ello, centraremos nuestros esfuerzos en destacar la relevancia evolutiva de un canal particular de herencia: la herencia epigenética. El trabajo se estructurará en cuatro secciones. En la primera sección se analizarán los principios generales de la Síntesis Moderna, haciendo hincapié en los conceptos de herencia y variación. En la segunda sección se analizarán las características más definitorias de la epigenética, resaltando los principales desafíos que esta disciplina establece a los conceptos clásicos de herencia y variación. En la tercera sección se analizará la pertinencia de integrar los fenómenos epigenéticos en los modelos evolutivos. De estos análisis se concluirá la necesidad de adoptar un pluralismo en lo relativo al concepto de herencia, una postura que diversos biólogos y filósofos han empezado a reivindicar (Bonduriansky y Day, 2009, 2018; Danchin et al., 2011, 2019; Mameli, 2004, 2005; Stotz y Griffiths, 2018; Uller y Helenterä, 2017). 


\section{LA Síntesis Evolutiva Moderna}

Los proponentes la Síntesis Moderna, apoyándose en los descubrimientos de la biología molecular, han asumido en sus escritos la existencia de un único mecanismo de herencia evolutivamente significativo: la transmisión genética. La presuposición de que la herencia no constituye otro fenómeno más que la transmisión de secuencias de ADN de ancestro a descendiente constituye uno de los principios clave que se encuentra tras los modelos poblacionales de la teoría evolutiva. Esta asunción genocentrista puede tomar dos formas (Mameli, 2004): una fuerte y otra débil. La primera asumiría que la transmisión genética constituye el único mecanismo de herencia. La segunda afirmaría que, si bien existen múltiples mecanismos de herencia, la transmisión genética constituye el único mecanismo hereditario relevante a nivel evolutivo. En el presente artículo se intentará poner en cuestión ambas versiones tomando la epigenética como fenómeno de referencia. ${ }^{1}$ Para ello, se sostendrá, en primer lugar, que existe una pluralidad irreductible de canales de herencia cualitativamente distintos entre sí, cuestionando, de esta manera, el sentido fuerte. En segundo lugar, que la transmisión genética no constituye el único mecanismo hereditario relevante a nivel evolutivo, cuestionando, así, el sentido débil.

Antes de continuar, es preciso aclarar el sentido mínimo bajo el cual se ha comprendido el fenómeno de la herencia. Definición mínima: la herencia abarca todos aquellos procesos o canales biológicos responsables del parecido o similitud entre los ancestros y los descendientes (Futuyma y Kirkpatrick, 2017, p. 106; Mameli, 2004; Ridley, 2004, p. 74). Es decir, todos aquellos procesos responsables de la recurrencia de las características fenotípicas en los linajes.

Teniendo en mente esta definición, los defensores de la Síntesis Moderna han sostenido que el único mecanismo de herencia capaz de causar una similitud relevante a nivel evolutivo es la transmisión genética (Ridley, 2004, p. 81). El motivo de ello es doble. En primer lugar, puesto que los determinantes últimos de los fenotipos son los genes y dado que estos se transmiten de los ancestros a los descendientes, la transmisión genética debe ser vista como el responsable último de la similitud fenotípica. Si la selección actúa fundamentalmente sobre la variación fenotípica hereditaria, esto es, sobre la variación codificada genéticamente, entonces el único mecanismo de herencia relevante a nivel evolutivo debe ser la transmisión genética. La evolución adaptativa debe ser vista, por tanto, en términos de cambios en las frecuencias genéticas. En segundo lugar, y en estrecha conexión con el punto

1 La inadecuación del sentido fuerte se abordará implícitamente a partir de las críticas al sentido débil. 
anterior, debido a la extraordinaria estabilidad y fidelidad con la que este mecanismo es capaz de transmitir la información de generación en generación (Herron y Freeman, 2015, p. 166). Se establece, así, un concepto de herencia puramente genético: el material hereditario y el material genético se convierten en elementos intercambiables (Helenterä y Uller, 2010). Tanto la estabilidad como la diferencia en los rasgos fenotípicos tiene su origen en el genoma mismo. Ninguna diferencia fenotípica que no esté codificada en el material genético puede ser heredada. Es por ello por lo que todo cambio somático inducido ambientalmente es incapaz de influir en los factores contenidos en la línea germinal (barrera de Weismann), careciendo de impacto sobre las dinámicas evolutivas (Mayr, 2001, p. 100).

Si bien es cierto que esta concepción genocentrista de la herencia se ha ido modificando hasta el punto de incluir fenómenos hereditarios no genéticos (endosimbióticos o citoplasmáticos), no es menos cierto que las tesis ontológica, epistemológica y metodológica- que subyacen a tal postura han permanecido, hasta cierto punto, inalteradas. La tesis ontológica sostendría que el organismo y el ambiente constituyen dos entidades independientes claramente diferenciadas. Es el medio interno el que sustenta el verdadero peso ontológico de la herencia. El ambiente es incapaz de ejercer ninguna influencia sobre el material genético del organismo (Herron y Freeman, 2015, p. 167). La tesis epistemológica afirmaría que el medio interno constituye una condición suficiente a nivel causal para dar cuenta de la estabilidad intergeneracional de los fenotipos. La tesis metodológica, por su parte, aseveraría que es preciso desacoplar el estudio de la herencia del estudio del desarrollo. Si bien las influencias ambientales constituyen un factor relevante en el estudio de la ontogenia de los organismos, estas carecen de importancia en el estudio de la herencia. ${ }^{2}$

La tesis ontológica $-y$ en cierta medida la metodológica ${ }^{3}$ - comete el error conceptual de tratar de separar el organismo de su ambiente (Lewontin,

2 El tratamiento de la herencia de Francesca Merlin ejemplifica a la perfección estas tres tesis: «the intergenerational transmisión, via reproduction, of the set of (genetic and nongenetic) factors and mechanisms conferring to the new entity the capacity to acquire (to have) the capacity to reproduce» (2017, p. 275). Si bien es cierto que la propuesta de Merlin reformula el concepto genocentrista de herencia hasta el punto de incluir factores citoplasmáticos o endosimbióticos, no es menos cierto que esta, a su vez, restringe la herencia a la mera transmisión de factores internos, subrayando, de esta manera, la dicotomía interno-externo. Esta consideración se hace sobre la base de la distinción herencia vs transmisión (aprendizaje social, efectos de exposición o efectos parentales). Para Merlin, los canales de transmisión, a diferencia de los de la herencia, serían incapaces de sostener la continuidad entre generaciones.

3 Decimos «en cierta medida» dado que la tesis metodológica puede ser justificada sobre la base de intereses pragmáticos. 
1998, p. 50). En la actualidad, se reconoce que los efectos ambientales constituyen un factor clave en la ontogenia de los organismos, dado que la expresión genotípica varia considerablemente en función de las condiciones ambientales. El ambiente (biótico y abiótico) juega, por tanto, un papel activo en la construcción de los fenotipos de los organismos (Sultan, 2015, p. 146). Tal y como indican Morris y Lundberg (2011), es imposible comprender la función de los rasgos y su influencia sobre la adecuación sin tener en cuenta el ambiente en cuestión, pues es precisamente este el que determina el curso particular de desarrollo que tomará un organismo, condicionando, de esta forma, su destino evolutivo. Esta relación es, sin embargo, de doble dirección. Diversos estudios (construcción del nicho) han demostrado que los organismos son capaces de alterar sus mismas condiciones de existencia pudiendo, de esta manera, modificar las presiones selectivas del ambiente (Odling-Smee et al., 2003; Sultan, 2015). Esta co-construcción del organismo y del ambiente pondría en cuestión la validez de la tesis ontológica $y$, en menor medida, la de la tesis metodológica. ${ }^{4}$

El problema con la tesis ontológica va más allá cuando somos conscientes de que el ambiente juega un papel clave en la producción de la variación fenotípica heredable. Uno de los principios básicos de la Síntesis Moderna es que los genes constituyen la única fuente de variación heredable, es decir, solo ellos proporcionan el material sobre el cual la selección puede actuar para alterar las dinámicas evolutivas de las poblaciones (Dawkins, 1999, p. 52). El único tipo de variación que demuestra tener un impacto sobre las dinámicas evolutivas es, por tanto, la variación genética, heredable y aleatoria. ${ }^{5}$ Esta fuente de variación tiene, fundamentalmente, dos orígenes: puede ser el resultado de combinaciones alélicas aleatorias generadas por medio de los mecanismos de reproducción sexual (Futuyma y Kirkpatrick, 2017, p. 85; Mayr, 2001, p. 108) o puede ser el resultado de cambios aleatorios en las secuencias de ADN -mutaciones-(Dobzhansky, 1970, p. 65). Estas consideraciones han sido recientemente puestas en duda a través de los recientes descubrimientos realizados en epigenética. Estos descubrimientos han revelado que es posible que nuevos fenotipos adaptativos puedan estar asociados a cambios epigenéticos que surgen en respuesta directa a los entornos ambientales, constituyendo, así, una fuente de variación heredable relevante a nivel evolutivo (Jablonka y Lamb, 2005).

4 Solo aceptando las influencias recíprocas que se dan entre los organismos y el ambiente es posible reconfigurar los diseños experimentales a fin de evaluar el alcance de los diversos canales de herencia existentes.

5 No son inducidas y no se correlacionan con el aumento de aptitud (Ridley, 2004, p. 88). 
Sobre la base de estas consideraciones, resulta sumamente difícil poder sostener la tesis epistemológica anteriormente mencionada. La herencia genética, por sí misma, es incapaz de dar cuenta, a nivel causal, del surgimiento y estabilidad intergeneracional de los diversos fenotipos. Las fuentes de desarrollo exogenéticas y los factores no genéticos influencian notablemente la conformación de los fenotipos intergeneracionalmente estables de la descendencia. Existen, por tanto, múltiples canales de información hereditaria cualitativamente distintos entre sí que divergen en lo relativo a su estabilidad, sensibilidad ambiental e impacto evolutivo. Esta multiplicidad de canales hereditarios cuestiona la suficiencia causal de la herencia genética (Jablonka y Lamb, 2005).

En este artículo se propondrá entender el concepto de herencia anteriormente enunciado de una manera inclusiva y pluralista: ${ }^{6}$ la herencia abarca todos aquellos procesos biológicos o canales de transmisión que contribuyan a la reconstrucción del fenotipo de la descendencia, esto es, a la recurrencia fenotípica dentro de los linajes. La herencia, y la misma evolución adaptativa, ya no descansa en un canal particular de herencia -la transmisión genética-, sino en todos aquellos mecanismos que contribuyan a la edificación y estabilidad de la similitud intergeneracional de los linajes (Uller y Helenterä, 2017). La adopción de este concepto lleva consigo dos implicaciones teóricas y dos correlatos prácticos. La primera implicación teórica es la siguiente: es necesario dejar de ver la transmisión genética como el mecanismo paradigmático de la herencia. Su correlato práctico es que es preciso estudiar la influencia y alcance de los diversos mecanismos no genéticos y exogenéticos que median en la reconstrucción de los fenotipos de la descendencia. La segunda implicación teórica es la siguiente: es preciso superar la dicotomía organismo-ambiente. Esto nos permitirá incorporar en el estudio de la herencia todos los resultados obtenidos por las disciplinas de la eco-devo y la construcción del nicho, logrando una mayor coherencia intrateórica dentro de la actual teoría evolutiva. El correlato práctico es que es preciso implementar una metodología pluralista y relacional en el estudio del fenómeno de la herencia, una metodología que permita entrever el alcance de los diversos canales de herencia, así como las interacciones que se establecen entre los mismos.

A fin de evaluar la viabilidad y validez de este concepto inclusivo de herencia, en este artículo nos centraremos en estudiar el alcance de los fenómenos epigenéticos hereditarios, así como su relación con la transmisión

6 Esta conceptualización se halla en línea con las consideraciones de Oyama, Griffiths y Gray (2001). 
genética y su impacto en la comprensión de las dinámicas evolutivas de las poblaciones.

\section{HaCIA UNA EXPANSIÓN DE LOS MARCOS DE LA SÍNTESIS MODERNA: LA EPIGENÉTICA}

La epigenética se ha definido como la rama de la biología responsable de estudiar los mecanismos moleculares que ocasionan cambios en la expresión genética, cambios que no involucran alteración alguna en las secuencias de ADN (Foust et al., 2015, p. 166). Los principales fenómenos epigenéticos estudiados han sido: 1. La metilación de la citosina en el carbono 5 del anillo de pirimidina (Bird, 2002), la cual conduce a la represión transcripcional. 2. Las modificaciones de las histonas, las cuales constituyen un conjunto de alteraciones (metilación, acetilación o fosforilación, entre otras) que se llevan a cabo en las regiones $\mathrm{N}$-terminales de las histonas. 3. Los ARN interferentes (ARNi), que constituyen moléculas de unos 20-25 nucleótidos de extensión que se caracterizan por inhibir la expresión genética (Hu y Barrett, 2017).

Es preciso identificar, desde un plano conceptual, qué tipo de variación epigenética es relevante para el concepto inclusivo de herencia propuesto en la anterior sección. Richards (2006) ha considerado que las variaciones epigenéticas pueden clasificarse en tres categorías en función de su asociación con la variación genética: obligatoria, facilitada y pura. La variación epigenética obligatoria se hallaría completamente determinada por la secuencia de ADN y seguiría las reglas de la genética mendeliana. Un ejemplo podría corresponder a la adquisición de los diferentes perfiles epigenéticos durante el proceso de diferenciación celular. La variación epigenética facilitada se hallaría parcialmente correlacionada con la variación genética, es decir, aquí el genotipo controlaría la variación epigenética de manera probabilística. Un ejemplo de esta clase de variación epigenética lo proporciona el estudio del ratón agouti (Morgan et al., 1999). Por su parte, la variación epigenética pura sería completamente independiente de la variación genética. Un claro ejemplo lo proporcionan los estudios sobre la divergencia de los patrones de metilación en los gemelos monocigóticos (Fraga et al., 2005). El tipo de variación relevante para el presente estudio es la variación epigenética pura -en menor grado la facilitada- dado que este tipo de variación se desacopla completamente de la variación genética, pudiendo suponer un sustrato de variación alternativo al genético.

En estrecha conexión con esta última caracterización, es conveniente distinguir entre las variaciones epigenéticas estocásticas -no dirigidas- de 
aquellas que son inducidas -dirigidas- por el medio ambiente ${ }^{7}$ (Vogt, 2017). Holliday definió a esta primera clase de variaciones como «epimutaciones»: cambios epigenéticos estocásticos que pueden surgir en respuesta a entornos estresantes -como tóxicos-, por errores en la reprogramación o por errores en la replicación del ADN (Cubas et al., 1999; Youngston y Whitelaw, 2008). ${ }^{8}$ Diversos estudios han reconocido la potencialidad que tienen los entornos estresantes para desencadenar tasas de epimutación más elevadas -tasas que son superiores a las genéticas (Lind y Spagopoulou, 2018). Si bien estas variaciones estocásticas pueden ser costosas en términos evolutivos, también pueden suponer una cobertura exitosa para aquellos organismos que se hallen inmersos en entornos desventajosos, para las especies que han colonizado un nuevo espacio adaptativo, para linajes asexuales o para organismos que tienen que hacer frente a cambios ambientales impredecibles y repentinos (Flatscher et al., 2012; Lind y Spagopoulou, 2018). El motivo es claro: las epimutaciones permiten explorar el espacio fenotípico, aumentando así las posibilidades de producir un fenotipo más adecuado (Pal y Miklos, 1999). A diferencia de las epimutaciones, las cuales se dicen estocásticas, ciertas variaciones epigenéticas tienen la potencialidad de responder a factores ambientales (Vogt, 2017). Se afirma que una variación epigenética está inducida por el medio ambiente cuando un estímulo exógeno al organismo ocasiona cambios epigenéticos dirigidos. De esta manera, es posible afirmar que las señales ambientales influyen en la conformación de fenotipos mediados epigenéticamente, ${ }^{9}$ algo que contraviene uno de los principios clave de la Síntesis Moderna: el carácter aleatorio de la variación. Este tipo de variación epigenética ha sido asociada tradicionalmente a la plasticidad fenotípica. No obstante, la relación entre estos dos fenómenos será un tema que se abordará en la sección IV. 1.2.

Varios autores han demostrado la posibilidad, no solo de que los cambios genéticos afecten a las tasas de variación epigenética, sino también que las mismas variaciones epigenéticas puedan afectar a la estabilidad misma del $\mathrm{ADN}$, ya sea alterando los ratios de mutación (mediante la desaminación espontánea), de recombinación (alterando la estructura de la cromatina) o afectando a la estabilidad de los elementos transponibles (Guerrero-Bosagna, 2012). Estas caracterizaciones muestran en qué medida la naturaleza de la variación es sumamente más compleja que la planteada por la Síntesis

7 Empero, ambas clases de variaciones son complementarias y no antagónicas (Leung et al, 2016).

8 Las epimutaciones no tienen porque estar siempre asociadas a cambios en la expresión genética (Hu y Barrett, 2017).

9 Un claro ejemplo lo constituyen las diferencias fenotípicas mediadas epigenéticamente que son inducidas por el régimen alimenticio -jalea real- entre las abejas melíferas reina y trabajadora (Lyko et al, 2010). 
Moderna: ya no se trata de que las variaciones genéticas puedan influenciar la generación de variaciones epigenéticas, las mismas variaciones epigenéticas pueden influenciar la generación de variaciones genéticas.

\section{III.1. LA HERENCIA EPIGENÉTICA}

Para que las variaciones epigenéticas puedan ser significativas en términos evolutivos es preciso demostrar que estas tienen la potencialidad de ser heredables (Heard y Martienssen, 2014). Y ya no solo eso, es preciso demostrar que estas son lo suficientemente estables como para que puedan ser seleccionadas, alterando así las frecuencias fenotípicas de las poblaciones (Jablonka, 2017).

En la actualidad, existe un amplio cuerpo de evidencia que avala la idea de que las marcas epigenéticas mediadas ambientalmente tienen la capacidad de ser transmitidas de generación en generación constituyendo un canal de información hereditaria alternativo al del ADN (Richards, 2006; Youngston y Whitelaw, 2008). Sin embargo, la literatura ha considerado que existen dos grandes objeciones que ponen en cuestión la validez e importancia evolutiva de la herencia epigenética:

1. La reprogramación de la línea germinal (Heard y Martienssen, 2014): el principal argumento en contra de la herencia epigenética procede de la eliminación de las marcas epigenéticas adquiridas durante la embriogénesis. La reprogramación de la línea germinal es un fenómeno de extraordinaria importancia ya que permite restaurar la totipotencia del cigoto evitando que las marcas epigenéticas interfieran con el correcto desarrollo del Bauplan. Es por este motivo por el que los críticos de la herencia epigenética han afirmado que esta se limita únicamente a una sola generación, por lo que no tiene la estabilidad suficiente como para constituir un mecanismo de herencia evolutivamente significativo (Boskovic y Rando, 2018). El motivo es claro: al no ser lo suficientemente estables, estas marcas epigenéticas no tienen la potencialidad de ser seleccionadas (Shea y Uller, 2011). Varios estudios, sin embargo, han demostrado que algunos loci tienen la potencialidad de escapar a esta reprogramación, sugiriendo que es posible transmitir la información epigenética de generación en generación vía línea germinal (Daxinger y Whitelaw, 2012; Jablonka y Raz, 2009; Sciamanna et al., 2019; Youngson y Whitelaw, 2008).

2. Confusión entre la herencia epigenética gamética y la no gamética (Heard y Martienssen, 2014): los críticos de la herencia epigenética afirman que los estudios que subrayan la plausibilidad de la misma suelen hacer referencia a casos de herencia epigenética no gamética, por lo que, de darse, el impacto de los fenómenos epigenéticos sobre las dinámicas evolutivas sería reducido. Desde esta perspectiva, se afirma que solo los casos de herencia 
epigenética gamética constituirían canales legítimos de herencia, pues solo ellos cuentan con una verdadera estabilidad transgeneracional. La herencia epigenética gamética constituye, de esta forma, el modelo paradigmático de la herencia epigenética transgeneracional. Tomando como punto de partida esta premisa, se afirma que la herencia epigenética transgeneracional tiene lugar cuando se cumplen las siguientes condiciones: 1. Condición de efecto: las marcas epigenéticas deben ocasionar alteraciones en la expresión genética, alteraciones que conduzcan, en última instancia, a diferencias fenotípicas. 2. Condición de línea germinal: las marcas epigenéticas deben ser transmitidas, en ausencia del estímulo ambiental, a través de la línea germinal. 3. Condición de estabilidad: las marcas epigenéticas deben persistir hasta la generación F3 en las hembras y F2 en los machos (Jirtle y Skinner, 2007; Perez y Lehner, 2019).

Esta conceptualización de la herencia epigenética transgeneracional es, empero, errónea desde un punto de vista teórico y práctico. Desde un punto de vista teórico, esta conceptualización parte de las tesis ontológica y epistemológica ya mencionadas en la sección II: es el medio interno el que sustenta el verdadero peso ontológico de la herencia y el que constituye la condición suficiente a nivel causal para dar cuenta de la estabilidad intergeneracional de los fenotipos. No obstante, ya vimos anteriormente que estas tesis adolecían de serias deficiencias teóricas y prácticas. Desde un punto de vista práctico, se ha demostrado que ciertos fenómenos de herencia epigenética no gamética pueden tener efectos transgeneracionales (condición de estabilidad). Es por ello por lo que aquí se propondrá renunciar a la condición de línea germinal, disociando, de esta manera, la herencia transgeneracional de la herencia epigenética gamética.

A fin de comprender esta segunda objeción y nuestra crítica a la misma, es preciso clarificar las características de los principales fenómenos de variación epigenética heredable. ${ }^{10}$ En primer lugar, tendríamos la herencia epigenética no gamética, que comprendería los efectos de exposición simultánea y los casos de transmisión independiente de la línea germinal. En segundo lugar, tendríamos los casos de herencia epigenética gamética. En los efectos de exposición simultánea, un mismo factor ambiental es capaz de afectar a la hembra embarazada (F0), al embrión en desarrollo (F1) y a la línea germinal del mismo ya diferenciada (F2) en el caso de las hembras, debido a que las células germinales del feto también pueden verse expuestas de forma directa (Cropley et al., 2006). Este primer caso no podría catalogarse estrictamente como «herencia epigenética gamética» pues la estabilidad del efecto epigenético se

10 Esta clasificación parte de las ideas de Danchin et al., (2019). Empero, aquí se han introducido modificaciones sustanciales, las cuales resumimos en Fig. 1. 
da por la exposición simultánea de varias generaciones a un mismo factor ambiental y tampoco como «herencia epigenética transgeneracional», dado que hasta el momento no se han reportado casos de la misma (condición de estabilidad). En la transmisión independiente de la línea germinal, diversas generaciones se ven expuestas a un mismo entorno recurrente -ambiental (Youngston y Whitelaw, 2008), parental (Badyaev, 2008; Badyaev y Uller, 2009) o cultural (Jablonka y Lamb, 2005)-, lo cual conduce a reconstruir en cada generación los patrones epigenéticos que conducen a los fenotipos alterados. Si las condiciones externas permanecen inalteradas, es posible que se dé una recurrencia en los fenotipos de los linajes en cuestión. En este caso, la estabilidad de las marcas epigenéticas será dependiente del contexto, es decir, constituiría un proceso «transgeneracional extrínseco». Aquí, si bien no se puede hablar de una «herencia epigenética gamética», podríamos hablar de «efectos epigenéticos transgeneracionales», efectos que podrían alterar las dinámicas evolutivas de las poblaciones. Se ha considerado, además, la plausibilidad de que estos fenotipos puedan, ulteriormente, estabilizarse y canalizarse mediante asimilación genética pudiendo responder a las presiones selectivas ${ }^{11}$ (Badyaev y Uller, 2009). En último lugar tendríamos la transmisión de línea germinal no genética, la cual podríamos asociar de manera directa con la «herencia epigenética gamética» y con los efectos «puramente transgeneracionales» (Anway et al., 2005; Guerrero-Bosagna et al., 2010). En este caso, los efectos ambientales o parentales alterarían directamente la línea germinal de tal forma que los efectos epigenéticos podrían persistir de generación en generación, siempre y cuando se diese una eliminación incompleta de las marcas epigenéticas durante la reprogramación de la línea germinal. De esta manera, las variantes epigenéticas podrían responder a las presiones selectivas (Danchin et al., 2011; Daxinger y Whitelaw, 2012; Richards, 2006; Wang et al., 2017).

Estos puntos demuestran en qué medida es preciso desacoplar los efectos epigenéticos transgeneracionales de la herencia epigenética gamética: algunos casos de herencia epigenética no gamética dan cuenta de la recurrencia fenotípica transgeneracional dentro de los linajes, cumpliendo con la definición inclusiva y pluralista de herencia propuesta en la sección II. En la siguiente sección trataremos de poner en cuestión la idea de que, para constituir un canal de herencia relevante a nivel evolutivo, los fenómenos epigenéticos deben ser estables transgeneracionalmente. Con ello demostraremos que incluso

11 Estas consideraciones pondrían en duda la distinción realizada por Merlin (2017) entre herencia y transmisión (ver nota 2). Lo que a su juicio constituyen simples canales de transmisión (efectos parentales o de exposición), a nuestro juicio constituyen canales de herencia, pues estos son capaces de dar cuenta de la continuidad entre generaciones.

Contrastes vol. XXVI-N³ (2021) 
los casos de herencia epigenética intergeneracional (F0-F1) cumplen con la definición inclusiva de herencia, pudiendo ser considerados como canales legítimos de herencia.

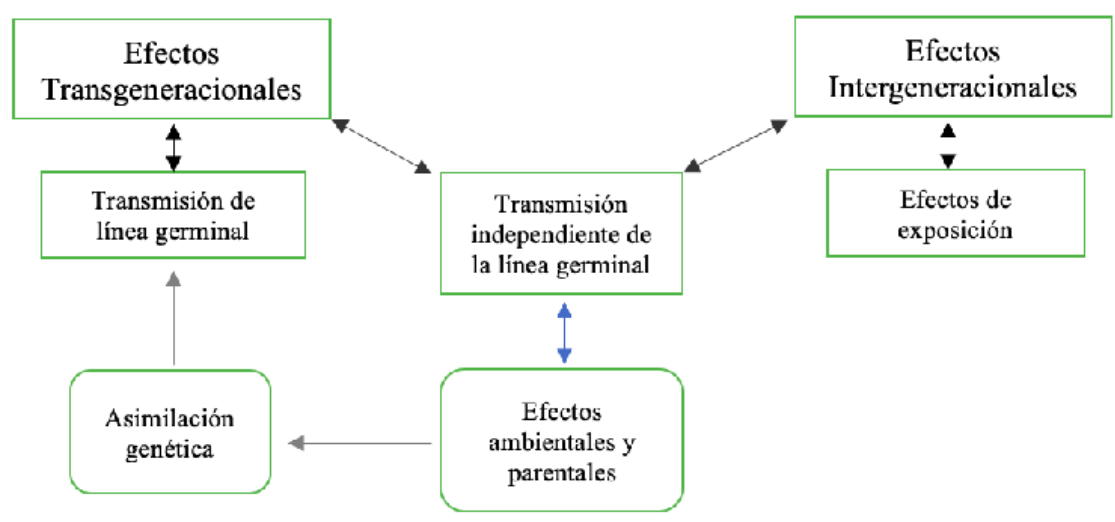

Fig. 1.

\section{EPIGENÉTICA Y EVOLUCIÓN}

Diversos autores han demostrado, ya sea mediante modelos teóricos (Bonduriansky y Day, 2018; Klironomos et al., 2013; Lind y Spagopoulou, 2018), cuantitativos (Banta y Richards, 2018) o experimentales (GuerreroBosagna et al., 2010; Morgan et al., 1999), que las variaciones epigenéticas son capaces de ejercer cierta influencia en las dinámicas evolutivas de las poblaciones.

Se ha señalado, empero, que para que los fenómenos epigenéticos puedan ser considerados relevantes a nivel evolutivo es preciso que estos cumplan con las siguientes condiciones:

1. Condición de efecto: Los estímulos ambientales deben inducir cambios en la expresión genética que resulten, en último término, en efectos fenotípicos.

2. Esta variación epigenética debe ser, si no completamente independiente de la variación genética, al menos parcialmente dependiente.

3. Los estímulos ambientales inductores deben acaecer en periodos clave de la ontogenia del organismo.

4. Condición de estabilidad: Estos cambios deben ser transmitidos a sucesivas generaciones, es decir, deben tener un efecto transgeneracional. 
5. Condición de línea germinal: Para tener un efecto transgeneracional, estos estímulos deben afectar, en último término, a las células de la línea germinal.

6. Esta variación epigenética debe responder a las presiones selectivas.

7. La acumulación de estos cambios fenotípicos mediados epigenéticamente afecta a la estabilidad evolutiva del linaje.

En las secciones anteriores, hemos analizado cómo las variaciones epigenéticas - pura o facilitada- eran capaces de desencadenar cambios heredables en la expresión genética en respuesta a señales ambientales. Estos cambios tenían la potencialidad de afectar a la conformación fenotípica pudiendo, en última medida, ser seleccionados (epialelos). Estas consideraciones han demostrado en qué medida es posible que la epigenética cumpla con los criterios 1-3 y 6 . También se estableció que era preciso disociar la herencia epigenética gamética de los efectos transgeneracionales, poniendo, así, en duda la legitimidad del punto 5. En la siguiente sección se analizarán los puntos 4 y 7. ${ }^{12}$ Solo de esta manera se podrá demostrar hasta qué punto la epigenética constituye un canal hereditario evolutivamente significativo, validando, así, el concepto de herencia inclusivo y pluralista que propusimos en la sección II.

\section{IV.1. LA EPIGENÉTICA Y LA ESPECIACIÓN}

Dentro del marco de la Síntesis Moderna, un aspecto fundamental de la evolución es la especiación, el proceso mediante el cual un grupo de organismos se separa del grueso de la población llegando a estar aislados en términos reproductivos de la especie ancestral. Desde el marco clásico, la dinámica de la especiación depende, en grado sumo, de un flujo genético restringido, así como de una presión selectiva diferencial. Son estos mecanismos los que, en último término, conducen a una incompatibilidad genética entre las poblaciones (Futuyma y Kirkpatrick, 2017, p. 220). No es de extrañar, por tanto, que la investigación evolutiva clásica se haya centrado en tratar de comprender, fundamentalmente, las causas genéticas -aislamiento reproductivo- y ecológicas - aislamiento geográfico- de la diversificación, obviando componentes tan esenciales como las dinámicas del proceso de desarrollo o los mismos mecanismos epigenéticos.

Para los defensores de la Síntesis Moderna, la especiación ocurre, principalmente, por accidente: las especies divergen debido a la aparición y ulterior propagación de mutaciones aleatorias. El aislamiento reproductivo

12 Es necesario recalcar que estos puntos, empero, se cumplen por los casos documentados de herencia epigenética gamética. 
evolucionaría de manera gradual como un mero efecto subsidiario. Teniendo en mente la metáfora del paisaje adaptativo de Wright -ese espacio multidimensional que representa las aptitudes medias de las poblaciones-el gran problema al que se ha tenido que enfrentar el pensamiento clásico es al siguiente: ¿cómo es posible que las especies puedan llevar a cabo la transición de un pico A a un pico B -éxito adaptativo- sin tener que atravesar el valle que conecta ambos picos, un valle que se define en términos de baja adecuación? Diversos autores han señalado que la epigenética es capaz de aportar una posible solución a esta laguna explicativa de los modelos poblacionales clásicos (Richards et al., 2010): los sistemas de herencia epigenética permiten que los organismos pasen de un pico adaptativo a otro evitando transitar los valles del paisaje adaptativo. Si bien la amplia mayoría de estudios evolutivos han afirmado que los cambios epigenéticos se siguen de los cambios genéticos (primera hipótesis de la especiación), existe una amplia evidencia que permite considerar la posibilidad de que los mecanismos epigenéticos sean la primera hipótesis teórica de la especiación (Vogt, 2017). En otras palabras, es posible que los genes sean los que sigan y no los que lideren los procesos de divergencia fenotípica y especiación (West-Eberhard, 2003, p. 158).

\section{IV.1.2. PLASTICIDAD, EPIGENÉTICA Y EVOLUCIÓN}

La plasticidad fenotípica constituye un fenómeno biológico de extraordinaria importancia, especialmente a la hora de dar cuenta del fenómeno de la especiación. La plasticidad se ha definido, tradicionalmente, como la capacidad que tiene un genotipo para producir diferentes fenotipos en respuesta a estímulos ambientales variables (Pfennig et al., 2010). Debido a su capacidad única para mediar la producción de rasgos fenotípicos sin variación genética subyacente- (West-Eberhard, 2003), la plasticidad tiene la potencialidad de mediar respuestas adaptativas directas, algo que es sumamente significativo en términos evolutivos. El motivo es claro: las respuestas plásticas pueden tanto disminuir los desajustes ambientales que acaecen a consecuencia del cambio repentino en las condiciones ecológicas como promover la colonización exitosa en entornos novedosos, motivando la radiación de las especies ${ }^{13}$ (Ehrenreich y Pfennig, 2016; Pfennig et al., 2010; West-Eberhard, 2003).

Recientemente, se ha demostrado que las variaciones epigenéticas inducidas ambientalmente pueden mediar estas respuestas plásticas adaptativas (Foust et al., 2015; Leung et al., 2016; Richards et al., 2010). Los efectos epigenéticos heredables podrían permitir, de esta forma, que los organismos

13 La plasticidad fenotípica es capaz de liberar variación genética críptica, la cual es expresada bajo cierto rango de condiciones ambientales o genéticas (Pfennig et al, 2010). 
se adaptasen y sobreviviesen al cambio en las condiciones ecológicas locales antes de que evolucionase la adaptación genética, esto es, antes de que se produzcan los cambios genéticos que codifican estos fenotipos ${ }^{14}$ (Pal y Miklos, 1999). La epigenética podría desempeñar, por tanto, un papel clave en las etapas iniciales de la especiación ecológica al mediar las dinámicas fenotípicas adaptativas de las poblaciones.

Una cuestión esencial que es preciso responder es la siguiente: ¿pueden los cambios epigenéticos contribuir de manera directa al aislamiento reproductivo? Diversos estudios han mostrado que la epigenética es capaz de conducir, ya sea indirecta o directamente, al aislamiento reproductivo de las poblaciones. Analicemos cada una de estas posibilidades. Indirectamente, al jugar un papel en la configuración de comportamientos mediados ambientalmente, la epigenética podría desempeñar un papel clave en el aislamiento reproductivo precruce - premating- (Gilbert y Epel, 2015, p. 447). Directamente, diversos estudios han señalado la posibilidad de que la epigenética pueda influir en el aislamiento reproductivo postcruce - postmating- de las poblaciones. ${ }^{15}$ Uno de los mecanismos propuestos es la alteración epigenética de la estructura de la cromatina. La compatibilidad epigenética es, por tanto, tan importante como la compatibilidad genética para garantizar la coherencia intraespecífica de las especies (Mendizabal et al., 2014).

Las variaciones epigenéticas podrían ejercer un mayor impacto en las dinámicas evolutivas de las poblaciones si estas pudiesen ser, en última medida, codificadas genéticamente (Ehrenreich y Pfennig, 2016). Waddington (1953) acuñó el término «asimilación genética» para hacer referencia al proceso por el cual rasgos que originalmente surgen en respuesta a factores ambientales -plasticidad- acaban codificándose de manera genética. Si el fenotipo generado por las respuestas plásticas mediadas epigenéticamente es adaptativo y lo suficientemente estable en el tiempo, entonces este persistirá en las poblaciones. Esas adaptaciones, en un principio epigenéticas, pueden tornarse permanentes si se produce un cambio de igual valor adaptativo en la secuencia del ADN. En último término, esa variación en la secuencia del ADN puede ser seleccionada, estabilizándose a un nivel genético (Gilbert y Epel, 2015, p. 464). Estas consideraciones nos permiten ver en qué medida la epigenética es capaz de satisfacer el requerimiento del punto 7 enunciado al comienzo de la sección.

14 Este podría ser el caso de la especie Viola cazorlensis estudiado por Herrera y Bazaga (2010).

15 Un ejemplo es la invasión del gorrión doméstico, Passer domesticus, en Kenia (Schrey et al, 2012). 
Si bien en la literatura acerca de la epigenética se suele dar por sentado que el único fenómeno evolutivamente significativo es la herencia epigenética transgeneracional -debido a su gran estabilidad-, diversos autores han señalado que incluso los sistemas de herencia epigenética de baja estabilidad -intergeneracionales- son capaces de afectar a las dinámicas evolutivas de las poblaciones a largo plazo (Danchin et al., 2019). No es preciso ni que el fenotipo heredado epigenéticamente goce de estabilidad transgeneracional ni que este acabe fijándose genéticamente por medio de la asimilación genética para que pueda ser relevante en términos evolutivos. Este hecho pondría en cuestión el punto 4 (condición de estabilidad) enunciado al comienzo de la sección. Las críticas a la relevancia evolutiva de los sistemas de herencia epigenética parecen sustentarse en la siguiente afirmación: para que sea evolutivamente significativo, dicen los críticos de la epigenética, el sistema de herencia epigenético debe ser cualitativamente similar al sistema de herencia genético. La cuestión central es que, teniendo en mente lo analizado hasta el presente momento, no es necesario, ni tampoco recomendable exigir que los sistemas de herencia epigenética cumplan con este requerimiento para que puedan ser significativos en términos evolutivos. Los motivos son los siguientes: 1. Tal y como se ha mencionado, los mecanismos epigenéticos, al mediar fenotipos adaptativos «inmediatos» en respuesta directa a las condiciones ambientales, permiten que las poblaciones subsistan aun cuando su aptitud genética es baja. Este hecho era particularmente relevante en el caso de las poblaciones con escasa variación genética y los linajes asexuales. 2. La inducción de variaciones epigenéticas estocásticas no adaptativas epimutaciones- puede ser relevante en términos evolutivos dado que estas permiten a las poblaciones explorar el espacio fenotípico, incrementando así la posibilidad de que estas puedan dar con un fenotipo que se encuentre más cerca del óptimo local (Pal y Miklos, 1999). 3. Los mecanismos epigenéticos son capaces de persistir, aun sin la consiguiente asimilación genética, en tanto en cuanto el estresor ambiental que los desencadena persista en el tiempo. 4. Tal y como se ha mencionado, una de las características definitorias de la epigenética es su reversibilidad. En cuanto el estímulo ambiental desaparece, la consiguiente respuesta fenotípica codificada epigenéticamente desaparece de igual manera. Este fenómeno puede tener cierto valor evolutivo. ¿Por qué motivo? Mientras que los cambios fenotípicos inducidos de manera genética dejan a las poblaciones «atrapadas» en el fenotipo que codifican -el cual puede ser costoso en términos evolutivos si las condiciones ambientales cambian drásticamente-, el fenotipo mediado epigenéticamente puede revertir a su estado previo si las condiciones ambientales inductoras desaparecen, otorgando a estos organismos una mayor ventaja adaptativa en caso de que las condiciones ambientales se modifiquen repentinamente (Burggren, 2016). 
5. En términos más generales, es posible afirmar que la herencia epigenética intergeneracional, al modificar la expresión fenotípica, es capaz de influenciar los resultados y la dirección evolutiva de las poblaciones, pues esta afecta de manera directa a la adecuación de los organismos al alterar su conformación fenotípica. Karola Stotz y Paul Griffiths (2017) han afirmado que el argumento (condición de estabilidad) de que la relevancia evolutiva de un sistema de herencia, en este caso la herencia epigenética intergeneracional, se mide en función de la estabilidad de sus marcas es non sequitur. La herencia epigenética intergeneracional, al modificar el fenotipo de la descendencia, es capaz de alterar el destino evolutivo de una población, puesto que la selección actúa sobre los fenotipos de los organismos. Es por este motivo por el que la herencia epigenética intergeneracional, a pesar de su relativa estabilidad, contribuye significativamente a la continuidad evolutiva de los linajes, cumpliendo con el requerimiento del concepto inclusivo y pluralista de herencia propuesto.

\section{HACIA UNA COMPRENSIÓN PLURALISTA E INCLUSIVA DE LA HERENCIA}

Comenzábamos este artículo señalando la pertinencia de adoptar un modelo pluralista e inclusivo de herencia. A lo largo del mismo se ha intentado hacer ver que la herencia constituye un fenómeno biológico sumamente complejo: consta de múltiples canales o procesos biológicos que contribuyen, de manera simultánea, a la estabilidad fenotípica de los linajes. Los investigadores, al decidir estudiar un canal particular de herencia -algo que está determinado por el proyecto explicativo y los intereses pragmáticos-, se abstraen de mucha de la complejidad subyacente a este fenómeno, ignorando la relevancia evolutiva de los restantes canales hereditarios: «success with one aim often inhibits success with other aims» (Potochnik, 2017, p. 105). Si bien es aceptable, desde un punto de vista explicativo, centrarse en el estudio de un canal particular de herencia -dados ciertos intereses de investigación-, es erróneo afirmar que exista un único mecanismo causalmente suficiente -la transmisión genética (Mameli, 2005). Este es el problema de fondo que se encuentra tras el debate en torno al concepto de herencia: la transmisión genética ha sido tomada como el modelo causal mínimo, aquel en el que se incluyen únicamente los factores que se cree que marcan la diferencia (Weisberg, 2013, p. 100). El error, en definitivas cuentas, ha consistido en tratar de equiparar la herencia a una de las múltiples abstracciones heurísticas posibles: la transmisión genética.

A lo largo del artículo, y tomando la epigenética como fenómeno de referencia, hemos dejado entrever dos razones principales por las que es conveniente considerar la plausibilidad de un modelo inclusivo de herencia: una negativa y otra positiva. La primera razón -negativa-, se debe a que la transmisión genética, por sí misma, es incapaz de dar cuenta de fenómenos tan relevantes como la fenogénesis, la recurrencia intergeneracional de los 
rasgos o la especiación. Es preciso, por tanto, adoptar este modelo integrador de herencia debido a la insuficiencia causal de los mecanismos genéticos de variación y herencia. La segunda razón -positiva-, se debe a que los fenómenos epigenéticos constituyen un nivel de explicación relevante, cualitativamente distinto e independiente de los fenómenos a nivel genético.

Cada uno de estos niveles de análisis -genético y epigenético- aborda una serie de problemas que le son propios y que sencillamente no pueden ser tratados desde el otro nivel de análisis. En este sentido, constituye una imposibilidad conceptual y práctica llevar a cabo una reducción de los fenómenos epigenéticos a los genéticos, y viceversa. Afirmar, sin embargo, que estos niveles son diferentes e irreductibles no implica asumir que las explicaciones aportadas por un nivel contradicen las aportadas por el otro. Es decir, no implica asumir que la epigenética y la genética constituyen hipótesis en competencia (Rosenberg, 2006). Las explicaciones aportadas desde la epigenética no contradicen las explicaciones genéticas dado que ambas disciplinas se ocupan de aspectos diferentes del fenómeno de la herencia. La inclusión de la epigenética lo que permite es, sencillamente, cubrir las lagunas explicativas que deja la genética. Cada canal hereditario juega, por tanto, un rol distintivo en el proceso evolutivo. Con esto no se está negando que exista cierta asimetría explicativa entre ambos niveles. Es posible que el nivel genético sea capaz de dar cuenta de una mayor variedad de aspectos, es decir, es posible que este disponga de un mayor alcance explicativo. Lo que se está afirmando es que ningún canal particular de herencia constituye una condición suficiente a nivel causal para dar cuenta de la fenogénesis, de la recurrencia intergeneracional de los fenotipos o del proceso de especiación. Reconocer la existencia de canales alternativos de herencia cualitativamente distintos entre sí constituye un paso teórico crucial: abre el camino a diseñar modelos experimentales que nos permitan comprobar el alcance y el impacto de los mismos.

Empero, sería erróneo creer que los múltiples canales de herencia operan de manera independiente los unos de los otros. La posibilidad de tal relación ha sido revisada considerando las posibles interacciones que pueden acaecer entre los canales epigenético y genético. No reconocer este último punto podría conducir a una suerte de aislacionismo que terminaría por oscurecer el mismo fenómeno a investigar (Mitchell, 2003). Es por ello por lo que una de las tareas a cumplir dentro del actual contexto de la EES es la de tratar de construir una visión unificada de los diversos mecanismos de herencia. Unificación en este contexto no significa reducción, no significa subsumir estos dos niveles de análisis bajo uno más general. Esto en sí mismo sería inviable dado que, como se ha mencionado, cada nivel abarca aspectos que el otro, por definición, es incapaz de abordar. Por unificación entendemos construir modelos teóricos, 
cuantitativos y experimentales que aborden los procesos biológicos teniendo en cuenta las particularidades y las interrelaciones que se establecen entre cada uno de estos canales de regulación.

\section{CONCLUSIÓN}

En el presente artículo se ha intentado defender, tomando como base la epigenética, la plausibilidad de un modelo pluralista e inclusivo de herencia, un modelo que se aleja conceptualmente de las pretensiones originarias de la Síntesis Moderna y que se muestra más en línea con la EES. Adoptar tal modelo pluralista de herencia permitirá superar muchas de las limitaciones inherentes a los sistemas de herencia genéticos, limitaciones que merman la posibilidad de lograr una comprensión más profunda de la evolución.

\section{REFERENCIAS BIBLIOGRÁFICAS}

ANWAY, M., CUPP, A., UZUMCU, M. Y SKINNER, M. 2005: «Epigenetic transgenerational actions of endocrine disruptors and male fertility». Science 308, pp. 1466-1469. Doi: https://doi.org/10.1126/science.1108190.

BADYAEV, A. 2008: «Maternal effects as generators of evolutionary change: A reassessment». Ann. N.Y. Acad. Sci. 1133, pp. 151-161. Doi: https://doi. org/10.1196/annals.1438.009.

BADYAEV, A. y ULLER, T. 2009: «Parental effects in ecology and evolution: mechanism, processes and implications». Philos. Trans. R. Soc. Lond. B. Biol. Sci. 364 (1520), pp. 1169-1177. Doi: https://dx.doi.org/10.1098\%2Frstb.2008.0302.

BANTA, J. y RICHARDS, C. 2018: «Quantitative epigenetics and evolution». Heredity 121 (3), pp. 210-224. Doi: https://doi.org/10.1038/s41437-018-0114-x.

BIRD, A. 2002: «DNA methylation patterns and epigenetic memory». Genes. Dev. 16, pp. 6-21. Doi: https://doi.org/10.1101/gad.947102.

BONDURIANSKY, R. y DAY, T. 2009: «Nongenetic inheritance and its evolutionary implications». Anu. Rev. Ecol. Evol. Syst. 40, pp. 103-125. Doi: https://doi. org/10.1111/jeb.12028.

BONDURIANSKY, R. y DAY, T. 2018: Extended Heredity. A New Understanding of Inheritance and Evolution. Princeton: Princeton University Press.

BOSKOVIC, A. y RANDO, O. 2018: «Transgenerational epigenetic inheritance». Annu. Rev. Genet. 52, pp. 21-41. Doi: https://doi.org/10.1146/annurevgenet-120417-031404.

BURGGREN, W. 2016: «Epigenetic inheritance and its role in evolutionary biology: re-evaluation and new perspectives». Biology 5 (24), pp. 1-22. Doi: https:// dx.doi.org/10.3390\%2Fbiology5020024.

CROPLEY, J., SUTER, C., BECKMAN, K. y MARTIN, D. 2006: «Germ-line epigenetic modification of the murine Avy allele by nutritional supplementation». PNAS 103 (46), pp. 17308-17312. Doi: https://doi.org/10.1073/pnas.0607090103. 
CUBAS, P., VINCENT, C. y COEN, E. 1999: «An epigenetic mutation responsible for natural variation in floral symmetry». Nature 401, pp. 157-161. Doi: https:// doi.org/10.1038/43657.

DANCHIN, É., CHARMENTIER, A., CHAMPAGNE, F., MESOUDI, A., PUJOL, B. y BLANCHET, S. 2011: «Beyond DNA: integrating inclusive inheritance into an extended theory of evolution». Nature 12, pp. 475-486.

DANCHIN, É., POCHEVILLE, A., REY, O., PUJOL, B. y BLANCHET, S. 2019: «Epigenetically facilitated mutational assimilations: epigenetics as a hub within the inclusive evolutionary synthesis». Biol. Rev 94, pp. 259-282. Doi: https://doi. org/10.1111/brv.12453.

DAWKINS, R. 1999: El fenotipo extendido. El largo alcance del gen. Madrid: Capitán Swing.

DAXINGER, L. y WHITELAW, E. 2012: «Understanding transgenerational epigenetic inheritance via the gametes in mammals». Nat. Rev. Genet. 13 (3), pp. 153-162.

DOBZHANSKY, T. 1970: Genetics of the Evolutionary Process. New York: Columbia University Press.

EHRENREICH, I. y PFENNIG, D. 2016: "Genetic assimilation: a review of its potential proximate causes and evolutionary consequences». Ann. Bot. 117 (5), pp. 769-779.

FLATSCHER, R., FRAJMAN, B., SCHÖNSWETTER, P. y PAUN, O. 2012 : «Environmental heterogeneity and phenotypic divergence: can heritable epigenetic variation aid speciation?» Genetics Research International. Doi: https://doi.org/10.1155/2012/698421.

FOUST, C., SCHREY, A. y RICHARDS, C. 2015: «Population Epigenetics». En O. Pontes y H. Jin. (eds), Nuclear functions in Plant Transcription, Signaling and Development. New York: Springer, pp. 165-179.

FRAGA, M. et al., 2005: «Epigenetic differences arise during the lifetime of monozygotic twins». PNAS 102 (30), pp. 10604-10609. Doi: https://doi. org/10.1073/pnas.0500398102.

FUTUYMA, D. y KIRKPATRICK, M. 2017: Evolution. Sunderland: Sinauer.

GILBERT, S. y EPEL, D. 2015: Ecological Developmental Biology: Integrating Epigenetics, Medicine, and Evolution. Sunderland: Sinauer.

GUERRERO-BOSAGNA, C. 2012: «Finalism in Darwinian and Lamarckian Evolution: lessons from epigenetics and developmental biology». Evol. Biol 39, pp. 283-300.

GUERRERO-BOSAGNA, C., SETTLES, M., LUCKER, B. y SKINNER, M. 2010: «Epigenetic transgenerational actions of vinclozolin on promoter regions of the sperm epigenome». PLoS ONE 5 (9), e13100. Doi: https://doi.org/10.1371/ journal.pone.0013100.

HEARD, E. y MARTIENSSEN, R. 2014: «Transgenerational epigenetic inheritance: myths and mechanisms». Cell 157, pp. 95-109. Doi: https://doi.org/10.1016/j. cell.2014.02.045. 
HELENTERÄ, H. y ULLER, T. 2010: «The Price Equation and Extended Inheritance». Philos. Theor. Biol. 2, pp. 1-17. Doi: http://dx.doi.org/10.3998/ ptb.6959004.0002.001.

HERRERA, C. y BAZAGA, P. 2010: «Epigenetic differentiation and relationship to adaptive genetic divergence in discrete populations of the violet Viola cazorlensis». New Phytol. 187, pp. 867-76. Doi: https://doi.org/10.1111/j.14698137.2010.03298.x.

HERRON, J. y FREEMAN, S. 2015: Evolutionary Analysis. England: Pearson.

HU, J. y BARRETT, R. 2017: «Epigenetics in natural animal populations». J. Evol. Biol. 30, pp. 1612-1632. Doi: https://doi.org/10.1111/jeb.13130.

HUNEMAN, P. y WALSH, D. (eds.) 2017: Challenging the Modern Synthesis. Adaptation, Development and Inheritance. Oxford: Oxford University Press.

HUXLEY, J. 1942/2010: Evolution: The Modern Synthesis. Cambridge: MIT Press.

JABLONKA, E. 2017: «The evolutionary implications of epigenetic inheritance». Interface focus 7, 20160135. Doi: https://doi.org/10.1098/rsfs.2016.0135.

JABLONKA, E. y LAMB, M. 2005: Evolution in Four Dimensions: Genetic, Epigenetic, Behavioral, and Symbolic Variation in the History of Life. Cambridge: MIT Press.

JABLONKA, E. y RAZ, G. 2009: «Transgenerational epigenetic inheritance: prevalence, mechanisms, and implications for the study of heredity and evolution». Q. Rev. Biol. 84 (2), pp. 131-176. Doi: https://doi.org/10.1086/598822.

JIRTLE, R. y SKINNER, M. 2007: «Environmental epigenomics and disease susceptibility». Nat. Rev. Genet 8 (4), pp. 253-262. Doi: https://doi.org/10.1038/ $\underline{\operatorname{nrg} 2045}$.

KLIRONOMOS, F., BERG, J. y COLLINS, S. 2013: «How epigenetic mutations can affect genetic evolution: model and mechanism». BioEssays 35, pp. 571578. Doi: https://doi.org/10.1002/bies.201200169.

LEUNG, C., BRETON, S. y ANGERS, B. 2016: «Facing environmental predictability with different sources of epigenetic variation». Ecology and Evolution 6 (15), pp. 5234-5245. Doi: https://doi.org/10.1002/ece3.2283.

LEWONTIN, R. 1998/2000: Genes, Organismo y Ambiente. Las relaciones de causa y efecto en biología. Barcelona: Gedisa.

LIND, M. y SPAGOPOULOU, F. 2018: «Evolutionary consequences of epigenetic inheritance». Heredity 121, pp. 205-209.

LYKO, F., FORET, S., KUCHARSKI, R, et al., 2011: «The honey bee epigenomes: differential methylation of brain DNA in queens and workers». PLoS Biol 8 (11). Doi: https://doi.org/10.1371/journal.pbio.1000506.

MAMELI, M. 2004: «Nongenetic Selection and Nongenetic Inheritance». Brit. J. Sci. 55, pp. 35-71. Doi: https://doi.org/10.1093/bjps/55.1.35.

MAMELI, M. 2005: «The inheritance of features». Biology and Philosophy 20, pp. 365-399.

MAYR, E. 2001: What evolution is. Great Britain: Phoenix paperback.

MENDIZABAL, I., KELLER, T., ZENG, J. Y SOOJIN, V. 2014: «Epigenetics and Evolution». Integrative and Comparative Biology 54 (1), pp. 31-42. Doi: https:// dx.doi.org/10.1093\%2Ficb\%2Ficu040. 
MERLIN, F. 2017: «Limited Extended Inheritance». En P. HunEMAN y D. WALSH. (eds.), Challenging the Modern Synthesis. Adaptation, Development and Inheritance. Oxford: Oxford University Press, pp. 263-280.

MITCHELL, S. 2003: Biological Complexity and Integrative Pluralism. Cambridge: Cambridge University Press.

MORGAN, H.D., SUTHERLAND, H. G., MARTIN, D. I. y WHITELAW, E. 1999: «Epigenetic inheritance at the agouti locus in the mouse». Nat. Genet. 23, pp. 314-318. Doi: https://doi.org/10.1038/15490.

MORRIS, D. y LUNDBERG, P. (2011): Pillars of Evolution. Fundamental Principles of the Eco-evolutionary Process. Oxford: Oxford University Press.

ODLING-SMEE, J., LALAND, K. y FELDMAN, M. (eds.) 2003: Niche Construction: The Neglected Process in Evolution. Princeton: Princeton University Press.

OYAMA, S., GRIFFITHS, P. y GRAY, R. (eds.) 2001: Cycles of Contingency. Developmental Systems and Evolution. Cambridge: MIT Press.

PAL, C. y MIKLÓS, I. 1999: «Epigenetic inheritance, genetic assimilation and speciation». J. Theor. Biol. 200, pp. 19-37. Doi: https://doi.org/10.1006/ jtbi.1999.0974.

PEREZ, M. y LEHNER, B. 2019: «Intergenerational and transgenerational epigenetic inheritance in animals». Nat. Cell. Biol. 21, pp. 143-151.

PFENNIG, D., WUNDT, M., SNELL-ROOD, E., CRUICKSHANK, T., SCHLICHTING, C. y MOCZEK, A. 2010: «Phenotypic plasticity’s impacts on diversification and speciation». Trends. Ecol. Evol. 25 (8), pp. 459-467. Doi: https://doi.org/10.1016/j.tree.2010.05.006.

PIGLIUCCI, M. 2007: «Do we need an extended evolutionary synthesis?». Evolution 61 (12), pp. 2743-2749. Doi: https://doi.org/10.1111/j.1558-5646.2007.00246.x.

PIGLIUCCI, M. y MÜLLER, G. (eds.) 2010: Evolution-The Extended Synthesis. Cambridge: MIT Press

POTOCHNIK, A. 2017: Idealization and the Aims of Science. Chicago: The University of Chicago Press.

RICHARDS, C., BOSSDORF, O. y PIGLIUCCI, M. 2010: «What role does heritable epigenetic variation play in phenotypic evolution?». BioScience 60 (3), pp. 232-327. Doi: https://doi.org/10.1525/bio.2010.60.3.9.

RICHARDS, E. 2006: «Inherited epigenetic variation - Revisiting soft inheritance». Nature reviews 7, pp. 395-401.

RIDLEY, M. 2004: Evolution. Oxford: Blackwell Publishing.

ROSENBERG, A. 2006: «Is Epigenetic Inheritance a Counterexample to the Central Dogma?». Hist. Phil. Sci. 28, pp. 549-566.

SCHREY, A., RICHARDS, C., MELLER, V., SOLLARS, V. y RUDEN, D. 2012: «The role of epigenetics in evolution: The extended synthesis». Genet. Res. Int. 2012, pp. 1-3. Doi: https://dx.doi.org/10.1155\%2F2012\%2F286164.

SCIAMANNA, I., SERAFINO, A., SHAPIRO, J. y SPADAFORA, C. 2019: «The active rol of spermatozoa in transgenerational inheritance». Proc. R. Soc. B 2086, pp. 20191263. Doi: https://doi.org/10.1098/rspb.2019.1263. 
SHEA, N., PEN, I. y ULLER, T. 2011: «Three epigenetic information channels and their different roles in evolution». J. Evol. Biol. 24, pp. 1178-1187. Doi: https:// doi.org/10.1111/j.1420-9101.2011.02235.x.

STOTZ, K. y GRIFFITHS, P. 2018: «Genetic, epigenetic and exogenetic information». En D. JoYCE. (ed.), Routledge Handbook of Evolution and Philosophy. London: Routledge.

SULTAN, S. 2015: Organism \& Environment. Ecological Development, Niche Construction and Adaptation. Oxford: Oxford University Press.

ULLER, T. y HELENTERÄ, H. 2017: «Heredity and Evolutionary Theory». En P. Huneman y D. Walsh. (eds.), Challenging the Modern Synthesis. Adaptation, Development and Inheritance. Oxford: Oxford University Press, pp. 280-317.

VOGT, G. 2017: «Facilitation of environmental adaptation and evolution by epigenetic phenotype variation: insights from clonal, invasive, polyploid, and domesticated animals». Environmental epigenetics 3 (1), pp. 1-17. Doi: https:// doi.org/10.1093/eep/dvx002.

WADDINGTON, C. 1953: «Genetic assimilation of an acquired character». Evolution 7, pp. 118-126. Doi: https://doi.org/10.1111/j.1558-5646.1953.tb00070.x.

WAGNER, G. y ALTENBERG, L. 1996: «Complex adaptations and the evolution of evolvability». Evolution 50, pp. 967-976.

WANG, Y., LIU, H. y SUN, Z. 2017: «Lamarck rises from his grave: parental environment-induced epigenetic inheritance in model organisms and humans». Biological Reviews 92, pp. 2084-2111. Doi: https://doi.org/10.1111/brv.12322.

WEISBERG, M. 2013: Simulation and Similarity. Using Models to Understand the World. Oxford: Oxford University Press.

WEST-EBERHARD, M. J. 2003: Developmental plasticity and evolution. England: Oxford University Press.

YOUNGSTON, N. y WHITELAW, E. 2008: «Transgenerational epigenetic effects». Annu. Rev. Genomics Hum. Genet. 9, pp. 233-257.

MARIANo Martín Villuendas es estudiante del doctorado "Lógica y Filosofía de la Ciencia" de la Universidad de Salamanca.

Lineas de investigación

Filosofía de la Ciencia y Filosofía de la Biología

Publicaciones recientes

(2021). «No somos nuestros genes: consideraciones en torno a la definición molecular de gen». Disputatio. Philosophical Research Bulletin 10 (16), pp. 103-137. Doihttps://doi.org/10.5281/zenodo.4678992

(2019): Una discusión en torno a los límites del concepto especie». Revista de Humanidades de Valparaíso 14, pp. 241-273. Doi:https://doi.org/10.22370/rhv2019iss14pp241-273

Correo electrónico: mmvilluendas@gmail.com 
